\title{
Adlayer Core-Level Shifts of Random Metal Overlayers on Transition-Metal Substrates
}

\author{
M. V. Ganduglia-Pirovano, ${ }^{1,2,3}$ J. Kudrnovský, ${ }^{4}$ and M. Scheffler ${ }^{1}$ \\ ${ }^{1}$ Fritz-Haber-Institut der Max-Planck-Gesellschaft, Faradayweg 4-6, D-14195 Berlin-Dahlem, Germany \\ ${ }^{2}$ Max Planck Institut für Physik komplexer Systeme, Außenstelle Stuttgart, Heisenbergstrasse 1, D-70569 Stuttgart, Germany \\ ${ }^{3}$ CAMP Department of Physics, Technical University of Denmark, DK-2800 Lyngby, Denmark \\ ${ }^{4}$ Institute of Physics, Academy of Sciences of the Czech Republic, CZ-18040 Prague 8, Czech Republic
}

(Received 3 July 1996)

\begin{abstract}
We calculate the difference of the ionization energies of a core electron of a surface alloy, i.e., a $B$ atom in a $A_{1-x} B_{x}$ overlayer on a fcc $B(001)$ substrate, and a core electron of the clean fcc $B(001)$ surface using density-functional theory. We analyze the initial-state contributions and the screening effects induced by the core hole, and study the influence of the alloy composition for a number of noble metaltransition metal systems. Our analysis clearly indicates the importance of final-state screening effects for the interpretation of measured core-level shifts. Calculated deviations from the initial-state trends are explained in terms of the change of inter- and intra-atomic screening upon alloying. A possible role of alloying on the chemical reactivity of metal surfaces is discussed. [S0031-9007(97)02582-9]
\end{abstract}

PACS numbers: 79.60.Dp, 73.20.At, 73.61.At

Surfaces of metallic alloys can act as efficient catalysts [1]. The yield of such chemical reactions depends sensitively on the structure and the composition of these surfaces. Recently, in trying to understand the role of the electronic interactions between the components of bimetallic catalysts, the shift of core-electron binding energies of surface atoms of metal adlayers on transitionmetal substrates have been studied, both experimentally [2] and theoretically [3-5]. It has been demonstrated that when final-state effects, i.e., contributions due to differences in final-state relaxation energies, do not modify the initial-state trend, core-level shifts can be used to deduce information on the adlayer's $d$ density of states (DOS) [3-5]. Indeed, for the studied adlayer systems, the shift of core-electron eigenvalues sensitively detected the shift of the valence $d$ band at the surface. The latter is of particular importance because the position of the surface $d$ band relative to the Fermi level is a key factor determining the chemical reactivity of the surface $[6,7]$.

In this Letter we extend these studies to random metallic overlayers on nonrandom metallic substrates (so-called surface alloys). In particular, we investigate the trends in variations of the core-level shifts of the surface atoms as a function of concentration of foreign atoms in the surface layer. We focus our attention on systems formed by the combination of transition and noble metals because of their different capabilities to screen the core hole created in the core-level photoemission spectroscopy. The study of the concentration dependence of the different contributions to the shifts allows for a deeper understanding of the physical mechanism underlying final-state effects on the core-level shifts at metal surfaces. We show that the trends in variations in these shifts are not always given by the initial-state shifts as in studies of nonalloyed surfaces $[5,8-10]$. We have identified two examples for which changes in the screening of the core hole due to modifica- tions of the chemical environment of the adatoms result in qualitatively different trends.

We present a density-functional theory study of the trends of the initial-state and final-state screening contributions to the adlayer core-level shifts for surface alloys. We define the adlayer core-level shift (ACLS) as the difference of the ionization energies of an adlayer coreelectron of a $B$ atom in a random overlayer $A_{1-x} B_{x}$ on a $B$ substrate and of a core electron of the clean surface of the $B$ metal. Thus, using the system $\mathrm{Cu}_{1-x} \mathrm{Pd}_{x} / \mathrm{Pd}(001)$ as an example, the ACLSs of a Pd atom are

$$
\begin{aligned}
\Delta_{\mathrm{ACLS}}^{\prime}= & \tilde{I}_{c}^{\mathrm{Cu}_{1-x} \mathrm{Pd}_{x} / \mathrm{Pd}(001)}-\tilde{I}_{c}^{\mathrm{Pd}(001)} \\
= & E^{\mathrm{Cu}_{1-x} \mathrm{Pd}_{x} / \operatorname{Pd}(001)}\left(n_{c}=1\right) \\
& -E^{\mathrm{Cu}_{1-x} \operatorname{Pd} \mathrm{Pd}_{x} / \operatorname{Pd}(001)}\left(n_{c}=2\right) \\
& -E^{\operatorname{Pd}(001)}\left(n_{c}=1\right)+E^{\operatorname{Pd}(001)}\left(n_{c}=2\right),
\end{aligned}
$$

where $\tilde{I}_{c}$ are the core-level ionization energies with respect to the Fermi energy, and $E$ are total energies of the ground state (two electrons in the core level, $n_{c}=2$ ) and the excited state $\left(n_{c}=1\right)$, calculated under the constraint of overall charge neutrality. The upper indices identify the considered systems, namely, the adsorbed $\mathrm{Cu}_{1-x} \mathrm{Pd}_{x}$ random monolayer on a $\operatorname{Pd}(001)$ substrate and the top layer of the clean $\operatorname{Pd}(001)$ surface. The prime in $\Delta_{\text {ACLS }}^{\prime}$ is to note that our definition here slightly differs from that introduced in Ref. [5]. In Ref. [5] admetal monolayers of $A$ atoms on a $B$ substrate were considered and the ionization energies of the $A$ atoms were compared with those of a clean surface of the $A$ crystal. Here, we calculate the difference of the ionization energies of a core electron of $B$ atoms in a $A_{1-x} B_{x}$ overlayer on a $B$ substrate and a core electron of a clean surface of the $B$ crystal. Thus, the full monolayer situation of $A$ on $B$ $(x \rightarrow 0)$ means the limit of zero concentration of $B$ atoms at random overlayer. 
Using the Slater-Janak transition-state concept to evaluate total-energy differences [11], we obtain from Eq. (1)

$$
\begin{aligned}
\Delta_{\mathrm{ACLS}}^{\prime} \approx & -\epsilon_{c}^{\mathrm{Cu}_{1-x} \mathrm{Pd}_{x} / \operatorname{Pd}(001)}\left(n_{c}=1.5\right) \\
& +\epsilon_{c}^{\operatorname{Pd}(001)}\left(n_{c}=1.5\right),
\end{aligned}
$$

where $\epsilon_{c}^{\mathrm{Cu}_{1-x} \mathrm{Pd}_{x} / \mathrm{Pd}(001)}$ and $\epsilon_{c}^{\mathrm{Pd}(001)}$ denote the Kohn-Sham eigenvalues of a particular core state of an adlayer Pd atom and a surface atom of clean $\operatorname{Pd}(001)$. In the initialstate approximation, the ACLSs are given by Eq. (2) with $n_{c}=2$.

The calculations of the ACLSs are performed by means of the surface Green's function technique based on the all-electron tight-binding linear muffin-tin orbital method (LMTO) within the atomic-sphere and the localdensity approximations (LDA) combined with the singlesite coherent potential approximation (CPA) in order to treat the effect of disorder [12]. Recently, the method has been successfully applied to the study of surface corelevel shifts (SCLSs) of the $4 d$ transition-metal surfaces [13]. A related approach has been applied to the study of the SCLSs of the $4 d$ and $5 d$ transition-metal surfaces [8]. Though they have included final-state effects because of the use of the frozen-core approximation, they are not able to separate initial and final-state contributions to the shifts. The present approach is ideally suited to the nature of the problem since the whole concentration range, from the monolayer to the single impurity limit, can be treated on an equal footing as can the neutral system and the system with a core hole at a single surface atom. The potentials are calculated self-consistently with respect to both CPA and LDA in a region consisting of the overlayer or the surface layer, three substrate layers, and two layers of empty spheres at the vacuum-sample interface. This region is coupled via the surface Green's function technique to the semi-infinite vacuum on one side and to the semi-infinite crystal on the other, with frozen potentials. An ideal epitaxial growth has been assumed that means that all interatomic distances in the overlayer, as well as between overlayer and substrate, are assumed to be the same and equal to that in the substrate. Calculations are performed for sphere radii chosen so as to minimize the total energy of the bulk substrate in the fcc structure. To describe the transition state [see Eq. (2)], separate LDA self-consistent calculations of a single impurity, with half an electron missing in the particular core level, at the random overlayer and at the clean surface are performed under the constraint of overall charge neutrality.

For the random overlayer case, the properties of the individual atoms occupying the ideal lattice sites are characterized by the coherent potential function matrix for the corresponding nonionized system. Strictly speaking, this is an approximation as we would have to perform the configurational averaging with the core-ionized atom fixed at a given lattice site at the random overlayer which would result in an inhomogeneous CPA medium centered around the impurity. Thus, the use of the calculated CPA medium that corresponds to the nonionized system with the same overlayer composition is the simplest meaningful approximation, which averages over the different configurations.

Figure 1(a) shows the calculated ACLS for $\mathrm{Cu} 2 p$ electrons in the random $\mathrm{Pd}_{1-x} \mathrm{Cu}_{x}$ overlayer on $\mathrm{Cu}(001)$ and Fig. 1(b) for $\mathrm{Pd} 3 d$ electrons in $\mathrm{Cu}_{1-x} \mathrm{Pd}_{x}$ on $\mathrm{Pd}(001)$. The results for $\mathrm{Ag} 3 d$ electrons in the random $\mathrm{Pd}_{1-x} \mathrm{Ag}_{x}$ overlayer on $\mathrm{Ag}(001)$ and for Pd $3 d$ electrons in $\mathrm{Ag}_{1-x} \mathrm{Pd}_{x}$ on $\mathrm{Pd}(001)$ are qualitative very similar to those of Figs. 1(a) and 1(b), respectively.

We will discuss first the trends of the core-electron eigenvalues of the nonexcited systems in their electronic ground states, i.e., the initial-state contributions to the ACLSs, $\Delta_{\mathrm{ACLS}}^{\text {initial }}$. The calculations show that the initialstate core-electron binding energies of $\mathrm{Cu}$ atoms in the $\mathrm{Pd}_{1-x} \mathrm{Cu}_{x}$ overlayer on $\mathrm{Cu}$ are lower than those of the surface atoms of the clean $\mathrm{Cu}$ surface, i.e., a negative initial-state shift [see Fig. 1(a)]. The magnitude of the shift increases with increasing foreign atom (Pd) concentration. Similar results are obtained for the $\mathrm{Ag}$ core electrons in $\mathrm{Pd}_{1-x} \mathrm{Ag}_{x}$ on $\mathrm{Ag}(001)$ relative to the clean $\operatorname{Ag}(001)$ surface. Also for the $3 d$ core electrons of $\mathrm{Pd}$ in $\mathrm{Cu}_{1-x} \mathrm{Pd}_{x}$ on $\mathrm{Pd}$ [see Fig. 1(b)] and in $\mathrm{Ag}_{1-x} \mathrm{Pd}_{x}$ on $\mathrm{Pd}$, we find that the initial-state shift relative to clean Pd surface is negative. Inspection of the surface $d$

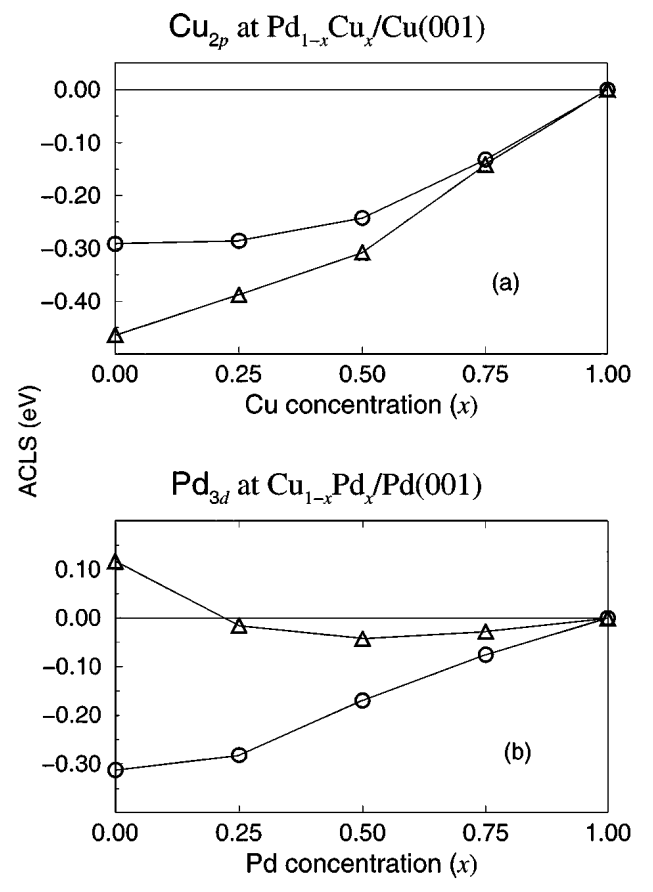

FIG. 1. (a) The ACLSs of $\mathrm{Cu} 2 p$ electrons in the random $\mathrm{Pd}_{1-x} \mathrm{Cu}_{x}$ overlayer on $\mathrm{Cu}(001)$ (triangles) [see Eq. (2)] and the initial-state contributions to the shifts (circles). The limit of zero concentration means a single $\mathrm{Cu}$ impurity in a $\mathrm{Pd}$ monolayer on $\mathrm{Cu}(001)$. (b) is similar to (a) but for the $\mathrm{Pd}$ $3 d$ electrons in $\mathrm{Cu}_{1-x} \mathrm{Pd}_{x}$ on $\operatorname{Pd}(001)$. 
DOS of the considered $B$ atoms in the random $A_{1-x} B_{x}$ overlayers shows for all four systems that the $d$ band width decreases with increasing concentration of foreign atoms, being narrowest for the single-impurity limit $(x \rightarrow$ 0 ). This result is indeed plausible and reflects the fact that coupling of $d$ orbitals between atoms is best when the neighbors are alike. Thus, the more distinct the neighbors are, the worse is the coupling and the stronger is the localization of the $d$ states. Figure 2(a) displays the $d$ contribution of the surface DOS of $\mathrm{Cu}$ atoms in the random $\mathrm{Pd}_{1-x} \mathrm{Cu}_{x}$ overlayer on $\mathrm{Cu}(001)$ for selected concentrations and Fig. 2(b) the corresponding result for $\mathrm{Pd}$ atoms in $\mathrm{Cu}_{1-x} \mathrm{Pd}_{x}$ on $\mathrm{Pd}(001)$. It is by now well established the essential correctness of the band narrowing argument [14], i.e., that a narrowing of the $d$ band of a surface atom is accompanied by a $d$ band shift in order to preserve $d$ band filling. The mentioned $d$ band-narrowing and shift to lower binding energies as the concentration of foreign atoms increases is clearly visible in Figs. 2(a) and 2(b). It should be noted that similarly to the case of clean surfaces [13] we have found a correlation between the initial-state contributions to the ACLSs and the shift of the $d$ band center as measured by the corresponding shift of the LMTO potential parameter $C_{d}$ [15], although deviations increase with the concentration of foreign atoms in the overlayer.

When final-state effects are taken into account, i.e., differences in the screening of the core hole by the other electrons, we find that the simple initial-state picture

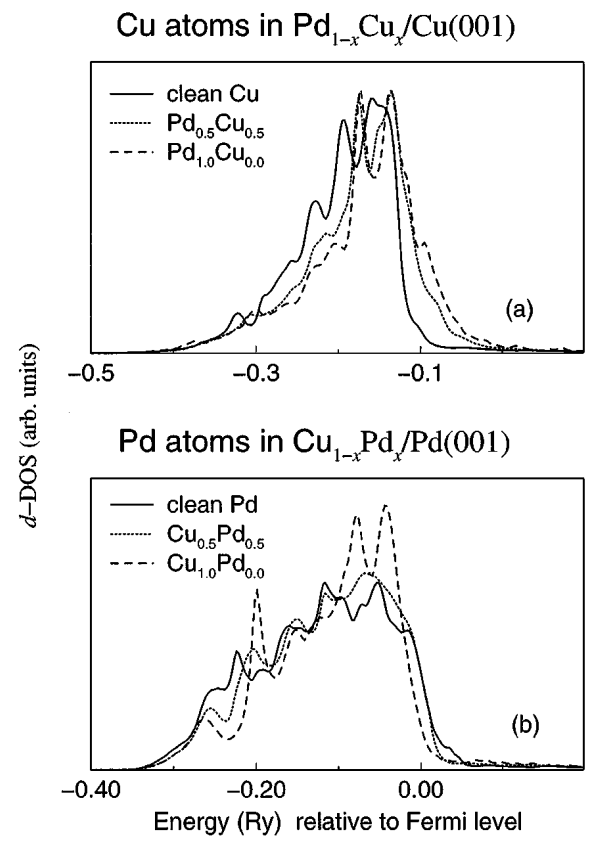

FIG. 2. (a) The $d$ DOS for $\mathrm{Cu}$ atoms in the random $\mathrm{Pd}_{1-x} \mathrm{Cu}_{x}$ overlayer on $\mathrm{Cu}(001)$ for selected concentrations. The limit of zero concentration means a single $\mathrm{Cu}$ impurity in a $\mathrm{Pd}$ monolayer on $\mathrm{Cu}(001)$. (b) is similar to (a) but for the $\mathrm{Pd}$ atoms in $\mathrm{Cu}_{1-x} \mathrm{Pd}_{x}$ on $\mathrm{Pd}(001)$. is changed considerably. Although it is often argued that such screening contributions are not very important (see, e.g., Refs. [3,16]) our explicit calculations clearly identify here the contrary. It has been already stressed that for clean surfaces and for pure metallic adlayers the screening contribution can amount to some tenths of an $\mathrm{eV}[5,8,9,17]$. Here, for the surface alloy case, we find that the contributions due to differences in screening of the core hole due to modifications of the chemical environment of the adatoms, can even change the qualitative trend of the initial-state approximation. The striking example is shown in Fig. 1(b).

Using the system $\mathrm{Cu}_{1-x} \mathrm{Pd}_{x} / \mathrm{Pd}(001)$ as an example, the screening contributions to the ACLSs of a Pd atom in the random $\mathrm{Cu}_{1-x} \mathrm{Pd}_{x}$ overlayer on $\mathrm{Pd}(001)$ are given by

$\Delta_{\text {ACLS }}^{\prime}-\Delta_{\text {ACLS }}^{\text {initial }}=\Delta \epsilon_{c}^{\mathrm{Cu}_{1-x} \operatorname{Pd}_{x} / \operatorname{Pd}(001)}-\Delta \epsilon_{c}^{\operatorname{Pd}(001)}$

where $\Delta \epsilon$ is the positive energy by which the core eigenvalue drops when half an electron is removed. A large positive screening contribution to the Pd $3 d$ ACLSs [see Fig. 1(b)] means that the Pd core levels in the random overlayer drop more strongly than at the clean surface when deoccupied. Thus, the $\mathrm{Pd} 3 d$ core hole is less well screened in the random $\mathrm{Cu}_{1-x} \mathrm{Pd}_{x}$ overlayer. A similar situation is found for $\mathrm{Pd}$ in $\operatorname{Ag}_{1-x} \mathrm{Pd}_{x}$ on $\operatorname{Pd}(001)$; i.e., the screening at the clean Pd surface is better than in the random $\mathrm{Ag}_{1-x} \mathrm{Pd}_{x}$ overlayer. On the other hand, for $\mathrm{Cu}$ in the random $\mathrm{Pd}_{1-x} \mathrm{Cu}_{x}$ overlayer on $\mathrm{Cu}(001)$ a negative screening contribution is obtained meaning that the $\mathrm{Cu}$ $2 p$ levels are better screened in the random overlayer [see Fig. 1(a)]. For $\mathrm{Ag}$ in $\mathrm{Pd}_{1-x} \mathrm{Ag}_{x}$ on $\mathrm{Ag}(001)$ a very small but positive contribution is obtained.

These results can be understood in terms of the explanations developed in Ref. [9] where it was noted that screening at surfaces of true (more than one $d$ hole) transition metals is better than in the bulk, whereas at surfaces of noble metals it is worse. Generalizing their analysis to the more complex situation of a surface alloy we conclude the following. The noticeably better screening of the $\mathrm{Pd} 3 d$ core hole at the clean $\mathrm{Pd}$ surface than in the random $\mathrm{Cu}_{1-x} \mathrm{Pd}_{x}$ and $\mathrm{Ag}_{1-x} \mathrm{Pd}_{x}$ overlayers is partially due to a worsening of the intra-atomic screening upon alloying, i.e., the screening provided by the localized $d$ states on the atom with the core hole. In fact, the $d$ DOS of the $\mathrm{Pd}$ atoms at the Fermi level decreases up to $60 \%(x \rightarrow 0)$ at the random overlayers. In addition, the interatomic screening, i.e., screening provided by surrounding atoms, also becomes worse upon alloying with noble metals such as $\mathrm{Cu}$ or $\mathrm{Ag}$. On the other hand, for noble metals such as $\mathrm{Cu}$ or Ag intra-atomic screening is less efficient due to the lower DOS at the Fermi level. Thus, interatomic screening becomes important, which is reflected by the better screening of the $\mathrm{Cu} 2 p$ hole in the random $\mathrm{Pd}_{1-x} \mathrm{Cu}_{x}$ overlayer as the $\mathrm{Pd}$ concentration increases. The finalstate screening contributions for $\mathrm{Ag}$ in $\mathrm{Pd}_{1-x} \mathrm{Ag}_{x}$ on $\mathrm{Ag}$ 
are very small but positive. Though one could have expected both $\mathrm{Cu}$ and $\mathrm{Ag}$ core holes to be equally screened when surrounded by $\mathrm{Pd}$ atoms, one should notice that $\mathrm{Pd}$ atoms in a $\mathrm{Cu}$ matrix as in $\mathrm{Pd}_{1-x} \mathrm{Cu}_{x} / \mathrm{Cu}(001)$ are considerably more compressed than in an $\mathrm{Ag}$ matrix (where interatomic distances are actually $5 \%$ larger that at a clean Pd surface). Thus, interatomic screening is facilitated in the first case. Inspection of the $\mathrm{Pd} d$ DOS of the random overlayers clearly shows the effect of the different hosts on the $\mathrm{Pd} d$ band, being much wider for $\mathrm{Pd}$ in $\mathrm{Pd}_{1-x} \mathrm{Cu}_{x}$ on $\mathrm{Cu}(001)$ than for the clean $\mathrm{Pd}(001)$ surface, which in turn is wider than for $\mathrm{Pd}$ in $\mathrm{Pd}_{1-x} \mathrm{Ag}_{x}$ on $\mathrm{Ag}(001)$.

In summary, the results presented here unambiguously show the importance of final-state screening effects for understanding and interpreting measured values of corelevel shifts. This conclusion agrees with some previous studies $[5,8-10,17]$ for ideal surfaces or admetal monolayers. In the present case, screening contributions originate from differences in the screening of the core hole due to modifications of the chemical environment of the overlayer atoms. The present calculations, which systematically separate initial- and final-state effects, show that the measured core-level shifts do not, in fact, necessarily correlate with the initial-state shifts, and therefore they do not necessarily reflect the shift of the surface $d$ DOS. The results support and stress the conclusion that the shift of the surface $d$ band is just an initial-state effect, and the use of measured values of ACLSs as an indication of the surface $d$ band shift is, in general, incorrect. We have also performed similar calculations for the $\mathrm{Cu}(\mathrm{Ni}) 2 p$ levels in the CuNi random overlayers on $\mathrm{Cu}(\mathrm{Ni})$. The difference in the efficiency of the screening between transition and noble metals is confirmed. Thus, the $\mathrm{Cu}(\mathrm{Ni}) 2 p$ core hole is better (worse) screened in the random CuNi overlayers than at the clean $\mathrm{Cu}(\mathrm{Ni})$ surface. The increased (negative) initial-state contributions to the ACLSs of Pd in the random overlayer of $\mathrm{Cu}_{1-x} \mathrm{Pd}_{x}$ on $\mathrm{Pd}(001)$ [see Fig. 1(b)] and in $\mathrm{Ag}_{1-x} \mathrm{Pd}_{x}$ on $\mathrm{Pd}(001)$ as the concentration of Pd decreases, which are accompanied by a higher Pd $d$ DOS closer to the Fermi level [see Fig. 2(b)], suggest that alloying an active metal like Pd with a catalytically inactive component, such as $\mathrm{Cu}$ or $\mathrm{Ag}$, would result in an increased chemical reactivity. Our predictions relate only to those changes in activity that can be interpreted in terms of an electronic effect and our above analysis would provide basic information on the influence of alloying on the surface reactivity only if the electronic effect is the dominant effect. It would be interesting to see if these predictions will be verified experimentally. Finally, we note that recently the variations in the surface electronic structure for supported monolayers as in Ref. [5] and substitutional impurities for a whole set of systems have been studied and similar predictions have been made [18].
M.V. Ganduglia-Pirovano thanks J. Norskov, A. Ruban, and M.H. Cohen for useful discussions. One of us (J. K.) acknowledges the financial support from the U.S.-Czechoslovak Science and Technology program (Project No. 95018). Center for Atomic-scale Materials Physics (CAMP) is sponsored by the Danish National Research Foundation.

[1] J.H. Sinfelt, Bimetallic Catalysts (Wiley, New York, 1983).

[2] J. A. Rodriguez, R. A. Campbell, and D. W. Goodman, J. Vac. Sci. Technol. A 10, 2540 (1992); Surf. Sci. 307309, 377 (1994).

[3] M. Weinert and R.E. Watson, Phys. Rev. B 51, 17168 (1995).

[4] R. Wu and A. J. Freeman, Phys. Rev. B 52, 12419 (1995).

[5] D. Hennig, M. V. Ganduglia-Pirovano, and M. Scheffler, Phys. Rev. B 53, 10344 (1996).

[6] B. Hammer and M. Scheffler, Phys. Rev. Lett. 74, 3487 (1995).

[7] B. Hammer and J. K. Norskov, Surf. Sci. 343, 211 (1995); B. Hammer, Y. Morikawa, and J. K. Norskov, Phys. Rev. Lett. 76, 2141 (1996).

[8] M. Alden, H. L. Skriver, and B. Johansson, Phys. Rev. Lett. 71, 2449 (1993); M. Alden, I. A. Abrikosov, B. Johansson, N. M. Rosengaard, and H. L. Skriver, Phys. Rev. B 50, 5131 (1994).

[9] M. Methfessel, D. Hennig, and M. Scheffler, Surf. Rev. Lett. 2, 197 (1995).

[10] E. Pehlke and M. Scheffler, Phys. Rev. Lett. 71, 2338 (1993).

[11] J.C. Slater, in Quantum Theory of Molecules and Solids (McGraw-Hill, New York, 1974), Vol. 4, pp. 51-55; J. F. Janak, Phys. Rev. B 18, 7165 (1978).

[12] J. Kudrnovský, I. Turek, V. Drchal, P. Weinberger, N.E. Christensen, and S. K. Bose, Phys. Rev. B 46, 4222 (1992).

[13] M. V. Ganduglia-Pirovano, V. Natoli, M. H. Cohen, J. Kudrnovský, and I. Turek, Phys. Rev. B 54, 8892 (1996).

[14] P. H. Citrin, G. K. Wertheim, and Y. Baer, Phys. Rev. Lett. 41, 1425 (1978); M.C. Desjonqueres et al., Solid State Commun. 34, 807 (1980); P.H. Citrin and G. K. Wertheim, Phys. Rev. B 27, 3176 (1983).

[15] H.L. Skriver, The LMTO Method (Springer-Verlag, Berlin, 1984).

[16] P. J. Feibelman and D. R. Hamman, Solid State Commun. 31, 413 (1979); Phys. Rev. B 28, 3092 (1983); P. J. Feibelman, Phys. Rev. B 39, 4866 (1989).

[17] J. R. Smith, F. J. Arlinghaus, and J. G. Gay, Phys. Rev. B 26, 1071 (1982).

[18] A. Ruban, B. Hammer, P. Stoltze, H. L. Skriver, and J. K. Norskov, J. Mol. Catal. A 115, 421 (1997). 